\title{
Erratum to: Marek's disease virus-encoded microRNAs: genomics, expression and function
}

\author{
LUO Jun ${ }^{1}$, TENG Man ${ }^{1}$, FAN JianMing ${ }^{1,2}$, WANG Fang Yu ${ }^{1}$, ZHOU Ling $^{1}$, \\ DENG RuiGuang ${ }^{1} \&$ ZHANG GaiPing ${ }^{1 *}$
}
${ }^{1}$ Key Laboratory of Animal Immunology of Ministry of Agriculture, Henan Provincial Key Laboratory of Animal Immunology, Henan Academy of Agricultural Sciences, Zhengzhou 450002, China;
${ }^{2}$ Laboratory of Toxicology, College of Public Health, Zhengzhou University, Zhengzhou 450001, China

\section{Erratum to: SCIENCE CHINA Life Sciences, October 2010 Vol.53 No.10: 1174-1180} doi: 10.1007/s11427-010-4073-6

In the original version of the article the fourth and fifth name above "Mid-cluster" in Figure 2 are incorrect. The correct names are M1-5p and M1-3p*.

The online version of the original article can be found at http://dx.doi.org/10.1007/s11427-010-4073-6 